\title{
LUMIO: achieving autonomous operations for Lunar exploration with a CubeSat
}

\author{
Stefano Speretta*, Angelo Cervone ${ }^{\dagger}$, Prem Sundaramoorthy ${ }^{\ddagger}$, Ron Noomen ${ }^{\dagger}$, \\ Samiksha Mestry ${ }^{\ddagger}$ and Ana Cipriano ${ }^{\S}$ \\ Delft University of Technology, Delft, 2629HS, The Netherlands
}

\author{
Francesco Topputo ${ }^{\mathbb{I}}$, James Biggs", Pierluigi Di Lizia ${ }^{\mathbb{I}}$, Mauro Massari ${ }^{\mathbb{I}}$, Kartik Mani**, \\ Diogene Dei Tos ${ }^{\dagger \dagger}$, Simone Ceccherini" ${ }^{\ddagger}$ and Vittorio Franzese** \\ Politecnico di Milano, Milano, 20156, Italy \\ Anton Ivanov $\$$ \\ École Polytechnique Fédérale de Lausanne, Lausanne, 1015, Switzerland \\ Demetrio Labate $\mathrm{e}^{\mathrm{IIII}}$ and Leonardo Tommasi**** \\ Leonardo, Campi Bisenzio, Firenze, 50013, Italy \\ Arnoud Jochemsen ${ }^{\dagger \dagger \dagger}$ and Janis Gailis \\ Science \& Technology AS, Oslo, Norway, 0160, Norway \\ Roberto Furfaro ${ }^{\S \S}$ and Vishnu Reddy \\ University of Arizona, Tucson, Arizona, 85721, USA \\ Johan Vennekens ${ }^{17}$ and Roger Walker ${ }^{17}$ \\ European Space Agency (ESA), European Space Research and Technology Centre, \\ Noordwijk, 2201 AZ, The Netherlands
}

The Lunar Meteoroid Impacts Observer (LUMIO) is one of the four projects selected within ESA's SysNova competition to develop a small satellite for scientific and technology demonstration purposes to be deployed by a mother ship around the Moon. The mission utilizes a 12U form-factor CubeSat which carries the LUMIO-Cam, an optical instrument capable of detecting light flashes in the visible spectrum to continuously monitor and process the meteoroids impacts. In this paper, we will describe the mission concept and focus on the performance of a novel navigation concept using Moon images taken as byproduct of the LUMIO-Cam operations. This new approach will considerably limit the operations burden on ground, aiming at autonomous orbit-attitude navigation and control. Furthermore, an efficient and autonomous strategy for collection, processing, categorization, and storage of

\footnotetext{
*Researcher, Faculty of Aerospace Engineering, Kluyverweg 1, 2629 HS , Delft - The Netherlands

${ }^{\dagger}$ Assistant Professor, Faculty of Aerospace Engineering, Kluyverweg 1, 2629 HS , Delft - The Netherlands

${ }^{\ddagger}$ Lecturer, Faculty of Aerospace Engineering, Kluyverweg 1, 2629 HS , Delft - The Netherlands

${ }^{\S}$ Student, Faculty of Aerospace Engineering, Kluyverweg 1, 2629 HS , Delft - The Netherlands

II Assistant Professor, Departement of Aerospace Science and Technology, Via La Masa 34, 20156, Milano, Italy, AIAA Member

"Associate Professor, Departement of Aerospace Science and Technology, Via La Masa 34, 20156, Milano, Italy, AIAA Member

** PhD student, Departement of Aerospace Science and Technology, Via La Masa 34, 20156, Milano, Italy

$\dagger$ Postdoc, Departement of Aerospace Science and Technology, Via La Masa 34, 20156, Milano, Italy

$\$$ Research Assistant, Departement of Aerospace Science and Technology, Via La Masa 34, 20156, Milano, Italy

$\S \S$ Director, Swiss Space Center, Route Cantonale, 1015, Lausanne, Switzerland

IIII Program Manager, Leonardo, Via delle Officine Galileo 1, 50013 Campi Bisenzio, Firenze, Italy

*** Systems Engineer, Leonardo, Via delle Officine Galileo 1, 50013 Campi Bisenzio, Firenze, Italy

${ }^{\dagger \dagger}$ Technical Manager, Tordenskioldsgate 6, 0160, Oslo, Norway

柿 Software Engineer, Tordenskioldsgate 6, 0160, Oslo, Norway

$\S \S \S$ Associate Professor, Departement of Systems and Industrial Engineering, University of Arizona, 1127 E. Roger Way, Tucson, AZ, 85721, USA

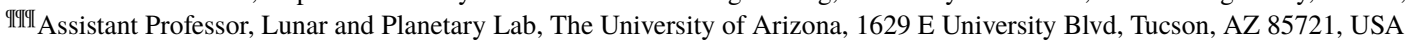

${ }^{17}$ Systems Engineer, Directorate of Technical \& Quality Management, Keplerlaan 1, 2201 AZ Noordwijk, The Netherlands
} 
payload data is also described to cope with the limited contact time and downlink bandwidth. Since all communications have to go via a Lunar Orbiter (mothership), all commands and telemetry/data will have to be forwarded to/from the mother ship. This will prevent quasi-real time operations and will be the first time for CubeSats as they have never flown so far from Earth.

\section{Introduction}

The Lunar Meteoroid Impact Observer (LUMIO) was one of the proposals submitted to the ESA SysNova LUnar CubeSats for Exploration (LUCE) call by ESA[1]. SysNova is intended to generate new and innovative concepts and to verify quickly their usefulness and feasibility via short concurrent studies. LUMIO was selected as one of the four concurrent studies run by ESA and it won ex-aequo the challenge. An independent assessment conducted at ESA's Concurrent Design Facility (CDF) has shown that the mission feasible, proving the values of LUMIO for future autonomous missions for planetary exploration.

The mission utilizes a CubeSat that carries the LUMIO-Cam, an optical instrument capable of detecting light flashes in the visible spectrum. On-board data processing is implemented to minimize data downlink, while still retaining relevant scientific data. The mission implements a sophisticated orbit design: LUMIO is placed on a halo orbit about Earth-Moon $\mathrm{L}_{2}$ where permanent full-disk observation of the Lunar farside is made. This prevents background noise due to Earthshine, and permits obtaining high-quality scientific products.

This paper will focus on the concept of operations, which will not have a direct communication link to Earth, preventing the usual navigation and control techniques. LUMIO will be especially relevant as a precursor or autonomous missions to remote bodies which cannot rely on real-time commands. Furthermore, in the optics of reducing the cost of a mission, operations (and navigation) will be autonomous, as operations is one of the cost figures that do not scale linearly with the satellite size[2].

In this paper we will present the mission (Section III), briefly describing also the SysNova LUCE challenge. We will then describe the orbit design (Section III) and concentrate on the mission concept of operations (Section IVP) and the autonomous navigation system (Section $\mathbf{V}$ ).

\section{Mission description}

LUMIO was one of the four competitive proposals selected for the ESA SysNova LUCE[1] study, which was aimed at identifying a viable low-cost concept using nano-satellites or CubeSats for interplanetary exploration. The LUCE call was, in particular, aimed at technology demonstration and the exploration of the Moon. The prize for this competitive study was the opportunity to review and advance the mission concept with ESA experts at the Concurrent Design Facility (CDF) at ESA/ESTEC.

\section{A. SysNova LUCE}

The LUCE study is expected to enable future exploration missions around the Moon, by pushing the following key technologies:

- Deployment and autonomous operation of a number of small satellites in a lunar orbit either as individual elements, or as part of a distributed system, including localization and navigation aspects;

- Miniaturization of optical, RF and other scientific payload instrumentation and associated technology flight demonstration on CubeSat/nano-satellite platforms in a lunar orbit;

- Remote sensing of the lunar surface, and/or in-situ measurements in the lunar environment and astronomical observations that could be made from lunar orbit and not achievable by past, current or planned lunar missions;

- Inter-satellite communication links to a larger Lunar Communications Orbiter for relay of data back to users on Earth and for tracking, telecommand and control;

- Technologies directly useful for future human and robotic exploration missions, and in need of flight demonstration in a representative environment.

The mission concept relays on a Lunar orbiter which departs from Earth and reaches an elliptical (800 km - $8000 \mathrm{~km})$ high-inclination orbit $\left(50^{\circ}-90^{\circ}\right)$ where it deploys several smaller satellites (up to $24 \mathrm{~kg}$ ) in a circular orbit around the Moon. This mother spacecraft solves most of the issues related to the deployment in Lunar orbit and it also ensures communication with Earth, acting as a relay to the small satellites (it should be noted that, according to the SysNova LUCE challenge, no direct-to-Earth communication was allowed). This concept brings several constraints on the small 


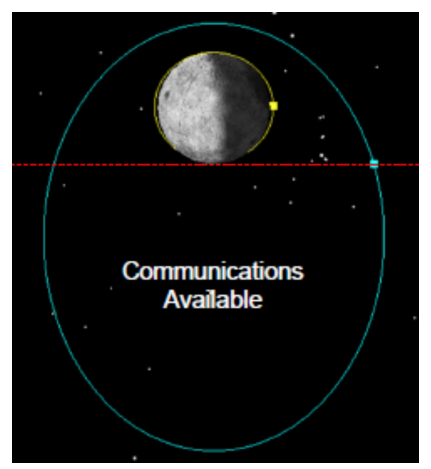

Fig. 1 Lunar Orbiter communication window

spacecrafts, especially from the communications point of view. The mother spacecraft is only visible in certain parts of the orbit (see Figure 1) and it constrains the communications to / from Earth as it can service only one satellite at the time in time-division multiple access. Furthermore, the mother spacecraft does not have a known schedule, so the deployed satellites should act independently and be able to fulfill their goals without counting on a connection to ground. An additional 10 days maximum communication blackout should also be considered, in case of problems onboard the mother spacecraft. One of the aims of SysNova LUCE is pushing the limits of technology and autonomous operations will be an important technology to demonstrate for future missions.

\section{B. Lunar Meteoroid Impact Observer}

LUMIO is one of the four missions that were funded by ESA and it was meant to observe, quantify, and characterize the meteoroid impacts by detecting the impact flashes on the lunar far side. This will complement the knowledge gathered by Earth-based observations of the lunar nearside, thus synthesizing a global information on the lunar meteoroid environment.

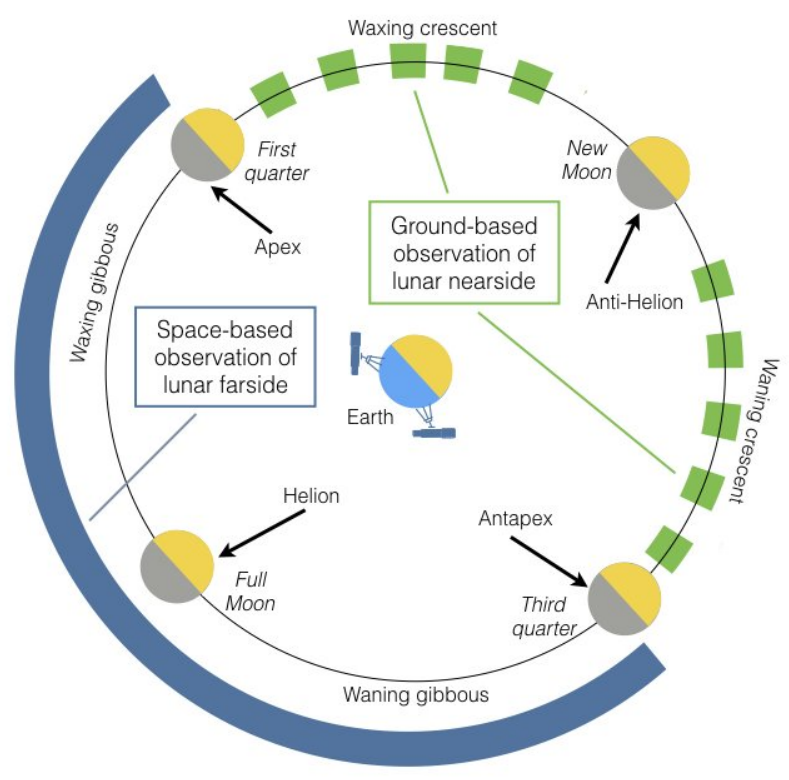

Fig. 2 Improvement in observation time of lunar impacts.

The mission is designed to observe meteoroid impacts on the lunar far side for a continuous period (up to 14 consecutive days) to improve the existing statistic on meteoroids close to Earth. The Moon can be used as an impact target to measure the statistic but Earth-based observations of lunar impact flashes are restricted to periods when the 
lunar nearside is illuminated between $10 \%-50 \%$. The observation on the night side of the Moon can be carried out when the illumination is less than $50 \%$ and this can happen for half of the lunar orbit. To achieve this, it was required to select an orbit that would maximize the visibility on the night side of the Moon (see Figure 2 for more details).

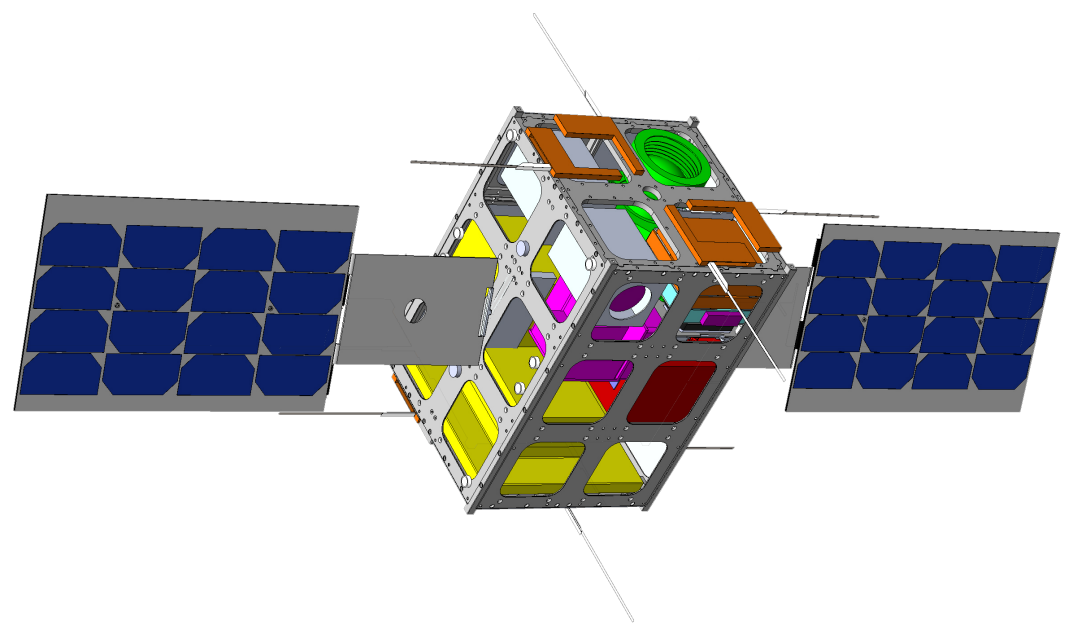

Fig. 3 LUMIO satellite configuration.

The mission uses a CubeSat that carries the LUMIO-Cam, an optical instrument capable of detecting light flashes in the visible spectrum. LUMIO-Cam has a $1024 \times 1024$ pixels CCD, $6^{\circ} \mathrm{FOV}, 127 \mathrm{~mm}$ focal length, and $55 \mathrm{~mm}$ aperture. Slight de-focusing is chosen to prevent detecting false positives. On-board data processing is implemented to minimize data downlink, while still retaining relevant scientific data. The on-board payload data processor autonomously detects flashes in the images, and only those containing events are stored.

The mission implements a sophisticated orbit design: LUMIO is placed on a halo orbit about Earth-Moon $\mathrm{L}_{2}$ where permanent full-disk observation of the lunar farside is made. This prevents having background noise due to Earth shine, and thus permits obtaining high-quality scientific products. Repetitive operations are foreseen, the orbit being in near 2:1 resonance with the Moon orbit. Innovative full-disk optical autonomous navigation is proposed, and its performances are assessed and quantified.

The spacecraft is a $12 \mathrm{U}$ form-factor CubeSat (see Figure 3 for further details), with a mass of $22 \mathrm{~kg}$. Novel on-board micro-propulsion system for orbital control, de-tumbling, and reaction wheel de-saturation is used. Steady solar power generation is achieved with a solar array drive assembly that also guarantees eclipse-free orbits. Accurate pointing is performed by using reaction wheels, IMU, start trackers, and fine sun sensors. Communication with the Lunar Orbiter is done in the UHF band using the CCSDS Proximity-1 link[10]. A lightweight structure with radiation shielding has been considered to minimize the impact of ionizing radiation on components, allowing to reduce mission cost by relying on commercial parts whenever possible.

To make such a mission possible, a propulsion system capable of a $\Delta v$ of $154 \mathrm{~m} / \mathrm{s}$ (see Section III)will be required. Several commercial units have been evaluated, deeming such system feasible, but requiring a high level of customization. The required volume, for such a system, has been estimated down to $3 \mathrm{U}$ with a wet mass of $5.6 \mathrm{~kg}$.

\section{Orbit}

A set of quasi-periodic halo orbits (sometimes referred here as quasi-halos or quasi-halo orbits) about Earth-Moon $\mathrm{L}_{2}$ are found by employing the methodology described in [11]. Fourteen quasi-halo orbits are computed in the high-fidelity roto-pulsating restricted n-body problem (RPRnBP) and saved as SPICE kernels. The initial feeds to compute the quasi-halo samples are Earth-Moon three-body halos at 14 different Jacobi constants, ranging from $\mathrm{C}_{j}=3.04$ to $\mathrm{C}_{j}=$ 3.1613263. All orbits are computed starting from 2020 August 30 00:00:00.00 TDB. Although quasi-halos, shown in Figure 4, are computed for a fixed initial epoch, the persistence of libration point orbits in the solar system ephemeris model allows wide freedom in the refinement algorithm, which also includes mission starting at different epochs[12].

Quasi-halo orbits of Figure 4 are all possible LUMIO operative orbits. As the orbit becomes more energetic (or as its CRTBP Jacobi constant decreases), the quasi-halo exhibits a wider range of motion both in terms of a) Moon range 

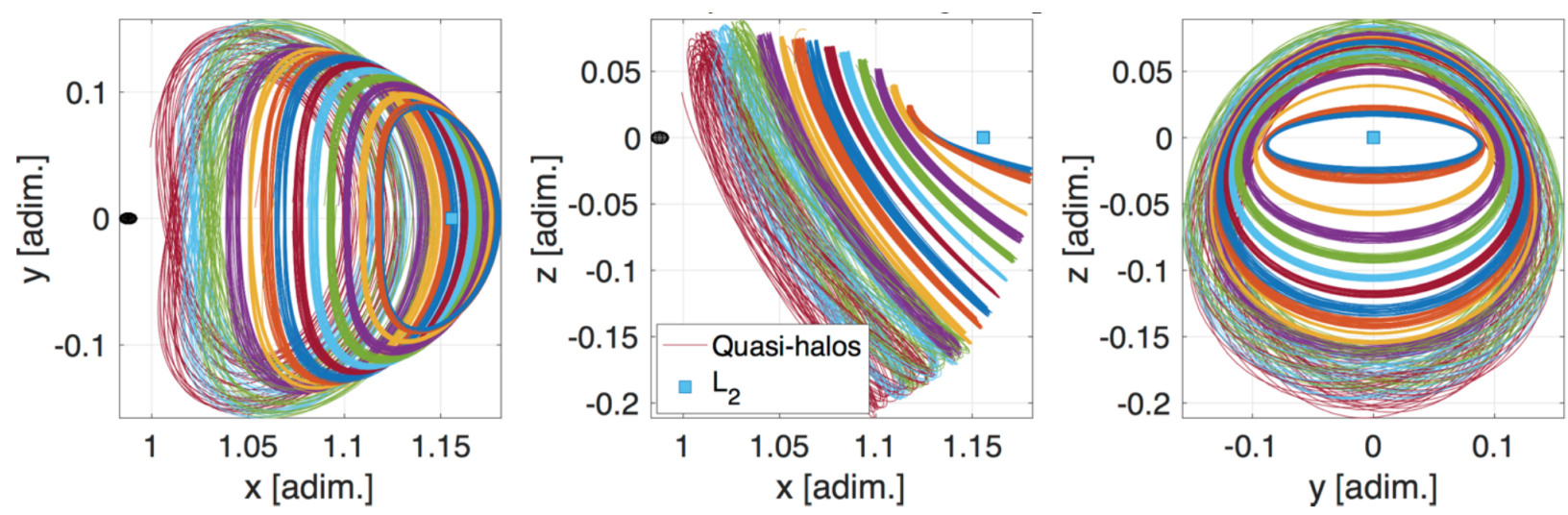

Fig. 4 Projection of Earth-Moon $L_{2}$ quasi-halos in the roto-pulsating frame.

and of b) geometrical flight envelope about the corresponding CRTBP trajectory. The latter trend is disadvantageous when a hard-pointing constraint must be respected (e.g., Moon full disk on optical instrument). On the other hand, the lunar distance places a constraint on the minimum FOV for the optical instrument on board LUMIO to be able to resolve the Moon full disk at any location along the quasi-halo.

The transfer phase of LUMIO is done entirely in the CRTBP. Free transport mechanisms are leveraged to reach a target halo. Specifically, intersection in the configuration space is sought between the halo stable manifolds and a selenocentric transition orbit. Since the sought intersection occurs only in configuration space, a maneuver is necessary for orbital continuity. This maneuver places the spacecraft on the stable manifold of the target halo and is thus called stable manifold injection maneuver (SMIM).

Mission $\Delta v$ budgets for each maneuver and phase are reported in Table 1 with both deterministic and confidence values. The $1 \sigma$ is $154.4 \mathrm{~m} / \mathrm{s}$, which is also in line with a $12 \mathrm{U}$ CubeSat volume and mass budgets. Note that ESA "Margin philosophy for science assessment studies" (Ref. SREPA/2011.097/, item MAT-DV-14) states that stochastic maneuvers shall be calculated based on the $3 \sigma$ confidence interval with no additional margins[13]. The choice to consider a $1 \sigma$ confidence interval on stochastic maneuvers for LUMIO is motivated by the inherently higher risk of a low-cost mission.

\begin{tabular}{|c|c|c|c|c|}
\hline \multirow{2}{*}{ Maneuver } & \multicolumn{4}{|c|}{ Cost $[\mathrm{m} / \mathrm{s}]$} \\
\hline & Deterministic & $1 \sigma$ & $2 \sigma$ & $3 \sigma$ \\
\hline PCM & 0 & - & - & - \\
\hline Transition orbit SK & - & 8 & 8 & 8 \\
\hline SMIM & 89.47 & - & - & - \\
\hline TCM1 & - & 28.6 & 53.0 & 73.1 \\
\hline TCM2 & - & 6.5 & 15.0 & 24.8 \\
\hline HIM & 0.5 & - & - & - \\
\hline 1-year SK & - & 18.3 & 23.9 & 28.1 \\
\hline Disposal & 3 & - & - & - \\
\hline TOTAL & \multicolumn{2}{|c|}{154.4} & 192.9 & 227.0 \\
\hline
\end{tabular}

Table 1 Mission $\Delta v$ budget.

\section{Concept of operations}

Autonomous operations is the key factor behind the design of LUMIO: the Lunar orbiter severely constrains the amount of information that can be sent and received and it also does not allow to plan operations in advance. Standard navigation, based on radiometric measurements with ground, is also impossible due to the lack of direct visibility. In 
our case, an autonomous navigation system (described further in Section $V$ ) has been designed to account for the lack of direct communication with Earth.

The start point of the LUMIO mission is the epoch of ejection from the Lunar Orbiter. The launch of the spacecraft aboard the launch vehicle and its trajectory from Earth to Moon is under the purview of the Lunar orbiter and it will not be described here. The mission is divided into four main phases, as also shown in Figure 5 a

- Parking orbit: this is the first phase of the mission, after deployment;

- Transit phase: during this phase, LUMIO will autonomously plan and execute a trajectory correction maneuver to reach the planned target orbit;

- Operational Phase: this is the nominal mission phase, when the science observations will be performed;

- End-of-life: in this phase LUMIO will be disposed to avoid risks for future missions.

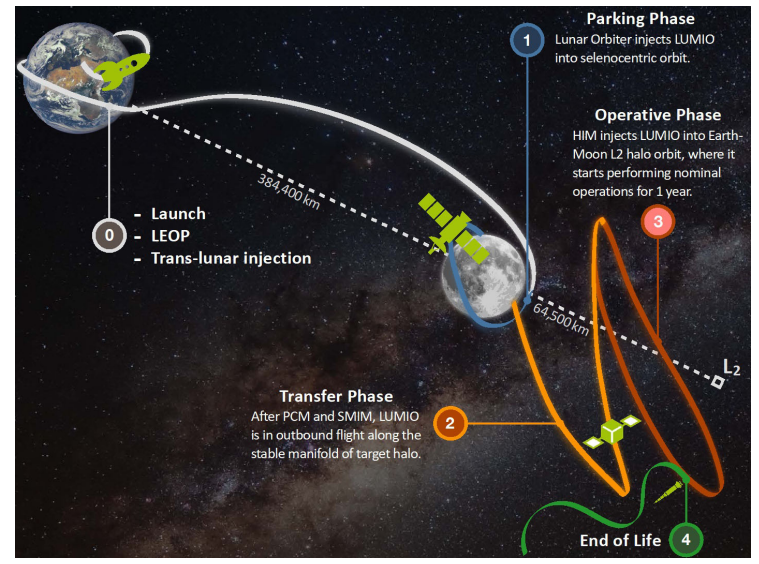

(a) Overall mission operational concept.

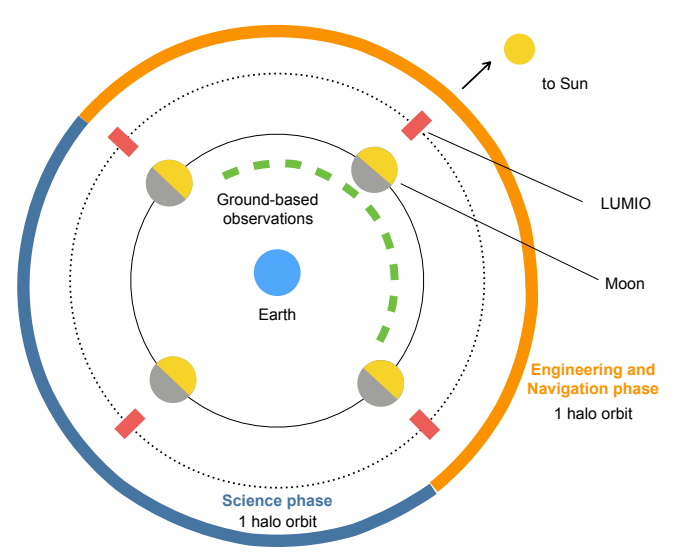

(b) Science operational concept.

Fig. 5 LUMIO operational concept.

\section{A. Parking Orbit}

This phase begins just after the orbiter releases LUMIO at the desired lunar parking orbit. In this phase, the first task is to commission the sub-systems that shall perform a status check for all to ensure their proper working. Preparations for the execution of the next phase are also done. It is important to stress here that the commissioning phase shall be completely independent (as opposed to most current CubeSat missions).

Once the proper working is ensured and the status of the systems are gathered, de-tumbling of LUMIO is performed to reduce the spin and stabilize it before the deployment of the solar arrays. This mode is important to start communications with the orbiter and initiate power generation. In case of commissioning failure, LUMIO will have to seek for help from Earth. Given the proposed injection orbit stability, minimal orbital corrections will be required for station keeping for approximately 14 days: this will guarantee enough time (considering a maximum 10 days communication blackout included in the original SysNova LUVE challenge) for proper control from ground, without impacting significantly the available fuel on-board.

After full deployment, LUMIO enters the cruise mode where the attitude control and navigation image acquisition will be done. For every revolution around the Moon, a station-keeping maneuver is performed to maintain the desired position and velocity.

\section{B. Transfer Phase}

Transfer trajectory optimization has been done for transfers from parking orbit to the quasi-halo orbit about L2 point considering the multi-body dynamics and minimization of propellant mass and time of flight. The analysis resulting from trajectory optimization yielded parameters that satisfy the mission requirements.

The transfer phase consists of four modes:

- Transfer Maneuver Mode: it contains three tasks, the Manifold Injection Maneuver, Trajectory Correction Maneuver ( $\times 2$ ), and Halo Injection Maneuver. The first one to inject LUMIO in a stable manifold to set it on 
course, the second one to correct the trajectory and reduce the deviation with the guidance, and the last one to insert LUMIO into a halo orbit.

- Transfer Cruise Mode: LUMIO cruises between every thrusting maneuver.

- Data Transmission: LUMIO communicates with the lunar orbiter and transmits the data.

- Desaturation: at the end of the phase, the reaction wheels are desaturated. Navigation acquisition, orbit and attitude determination are done to ensure the correct positioning of LUMIO. This is necessary to commence the operational phase of the mission.

The navigation system is responsible determining the proper time for the trajectory correction maneuvers to reach the desired science orbit: the main rationale for this selection is the possible absence of contacts to ground (always via the Lunar orbiter) just before the trajectory correction maneuver.

\section{Operational Phase}

The most important phase of the mission is the operational phase. The duration of this phase is 1 year and it has two main modes: Science Mode and Navigation \& Engineering Mode. The two modes occur within one Synodic Period ( 29.53 days) and are equally split with 14.765 days each. The operations are duty cycled and in 1 year, we have 12.3 cycles of Science and Navigation \& Engineering modes alternating between each other. This is shown clearly in Figure $5 b$.

Before entering one of these modes, there is a need to perform a commissioning of the payload data processor for science operations and payload instrument calibration to ensure its functionality and its readiness for meteoroid impacts observation.

\section{End-of-Life Disposal Phase}

At the end of the operational phase, LUMIO needs to be de-commissioned and disposed safely. The first step is to transmit the last pieces of data to the orbiter. All correction maneuvers are halted and the batteries are discharged until a small fraction of the energy required for EOL disposal maneuver. Finally, the propulsion system thrusts to execute the EOL disposal maneuver. Since we are in the early stages of mission development, the requirements regarding the environmental safety are still in development. The current strategy is leaving the Earth-Moon system as there would be a significant risk to impact future missions.

\section{Autonomous navigation}

One of the enabling technologies for LUMIO and other missions is autonomous navigation. Traditionally, spacecraft orbit determination is performed via radiometric tracking, but this implies the costs related to the ground segment, which do not scale down with mission size as other costs. Therefore, autonomous navigation is required for small spacecraft, and demonstrating this capability is a major objective of LUMIO[9]. Autonomous navigation will also allow to drastically reduce the operational cost and complexity of this mission, as it will be described in Section IV

\section{A. LUMIO Navigation Requirements}

The navigation subsystem requirements for the LUMIO mission are listed in Table 2 , The requirement NAV.001 comes from the ESA SysNova statement of work which demands for an autonomous on-board navigation system to be tested on a CubeSat. The accuracy needed for the position and velocity estimations as function of the mission phase, due to payload, mission analysis and S/K constraints, are stated in the requirements NAV.002-005. Lastly, NAV.006 imposes a higher bound for the state update frequency, to better manage the CubeSat on-board data processing for navigation purposes.

\section{B. Navigation Techniques Trade-Off}

Table 3 trade-offs navigation techniques about autonomy, accuracy, sensor technology, and cost for LUMIO. The demand of autonomous navigation excludes the Earth-based radiometric navigation for LUMIO. Autonomous navigation options are then the X-ray Pulsar navigation (X-NAV)[3], the Celestial Triangulation [4], and the Horizon-Based navigation [5]. The X-NAV, which performs spacecraft positioning by processing pulsars signals, is affected by sensor miniaturization difficulties. The Celestial Triangulation technique estimates a spacecraft position by triangulating with some observed celestial objects with known ephemeris, but is not compliant with LUMIO navigation requirements. The 
Table 2 Navigation requirements for LUMIO mission.

\begin{tabular}{|c|c|}
\hline ID & Requirement \\
\hline NAV.001 & The system shall perform autonomous on-board navigation. \\
\hline NAV.002 & $\begin{array}{l}\text { The navigation system shall determine the components of the satellite position vector within a } 30 \mathrm{~km} \\
\text { accuracy during engineering operations phase. }\end{array}$ \\
\hline NAV.003 & $\begin{array}{l}\text { The navigation system shall determine the components of the satellite velocity vector within a } 0.5 \mathrm{~m} / \mathrm{s} \\
\text { accuracy during engineering operations phase. }\end{array}$ \\
\hline NAV.004 & $\begin{array}{l}\text { The navigation system shall determine the components of the satellite position vector within a } 30 \mathrm{~km} \\
\text { accuracy, } 24 \text { hours before the station keeping maneuver execution in any phase. }\end{array}$ \\
\hline NAV.005 & $\begin{array}{l}\text { The navigation system shall determine the components of the satellite velocity vector within a } 0.3 \mathrm{~m} / \mathrm{s} \\
\text { accuracy, } 24 \text { hours before the station keeping maneuver execution in any phase. }\end{array}$ \\
\hline NAV.006 & The state update frequency shall be equal or lower than 1 update/min. \\
\hline
\end{tabular}

Horizon-Based navigation directly uses the Moon full disk images to estimate the spacecraft position. This is achieved by detecting the Moon full disk in an image and linking the Moon apparent size with the real one to estimate the relative distance. The full position vector can be estimated provided that Moon ephemeris and LUMIO attitude are known. The Horizon-Based navigation is the baseline option for LUMIO.

Table 3 Navigation techniques trade-off for LUMIO mission.

\begin{tabular}{lcccc}
\hline \hline & Autonomy & Accuracy & Sensor & Cost \\
\hline Radiometric Tracking & $\boldsymbol{N}$ & Order of meters & $\checkmark$ & $\boldsymbol{X}$ \\
Pulsar Navigation & $\checkmark$ & Order of km & $x$ & $\sim$ \\
Celestial Triangulation & $\checkmark$ & Order of $10^{3} \mathrm{~km}$ & $\checkmark$ & $\checkmark$ \\
Horizon-Based Nav. & $\checkmark$ & Order of $10^{2} \mathrm{~km}$ & $\checkmark$ & $\checkmark$ \\
\hline \hline & green Compliant yellow Adaptable & red Unacceptable
\end{tabular}

\section{Simulator}

The images acquired by the LUMIO-Cam are simulated employing POV-Ray F $^{\text {a }}$ a rendering software which generates synthetic Moon images as function of a camera position, orientation and properties. Figure 6 reports the simulator architecture. In the MATLAB working environment, the SPICE ${ }^{\dagger}$ toolkit is employed to set the LUMIO-Moon-Sun orbital geometry and the LUMIO attitude. These data are sent to POV-Ray, where properties belonging the to LUMIO-Cam are present, and a Moon image can be rendered.

\section{Horizon-Based Navigation}

The horizon-based optical navigation[5] employs the full-disk view of a spherical or ellipsoidal object to estimate the relative camera-to-object position vector. This is achieved by detecting the object full-disk in an image and linking the apparent object size in pixels with the real one. The image processing steps are shown in Figure 7. Once a Moon image is acquired (1), its edge is detected (2) through image processing algorithms (e.g., Canny edge detection [8]), and an ellipse if fit to the observed horizon points (3). The ellipse is an estimation of the object full-disk, and feeds the horizon-based optical navigation algorithm.

\footnotetext{
*Persistence of Vision Raytracer (POV-Ray), Williamstown, Victoria, Australia. Retrieved from http://www.povray.org.

†SPICE is NASA’s Observation Geometry and Information System for Space Science Missions [6. 7]. https://naif.jpl.nasa.gov/naif
} 


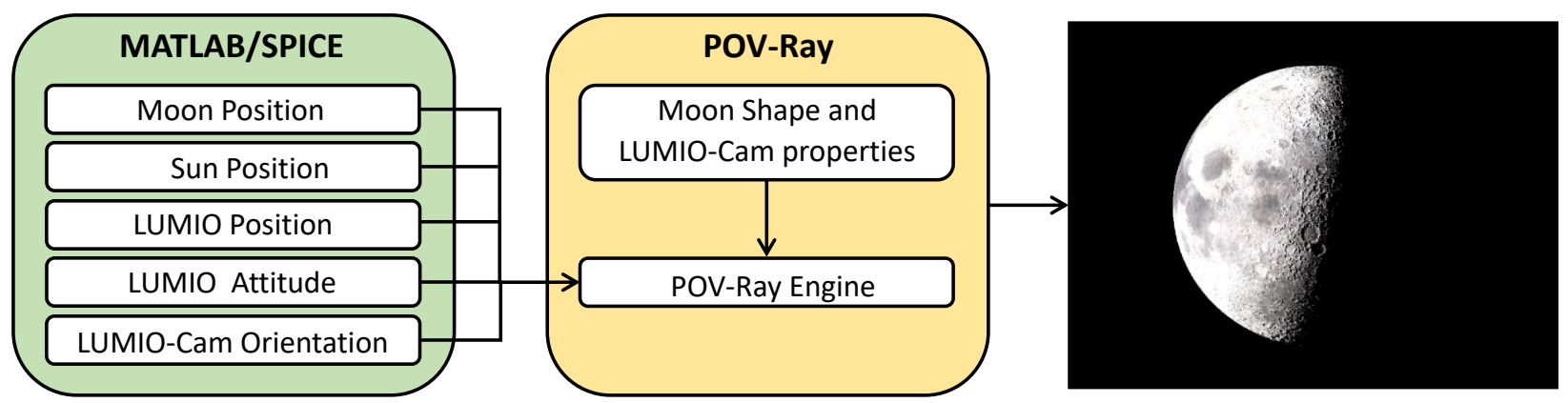

Fig. 6 Image generation process. The LUMIO-Moon-Sun geometry is defined in SPICE kernels, which is used in conjunction to POV-Ray to render Moon images.
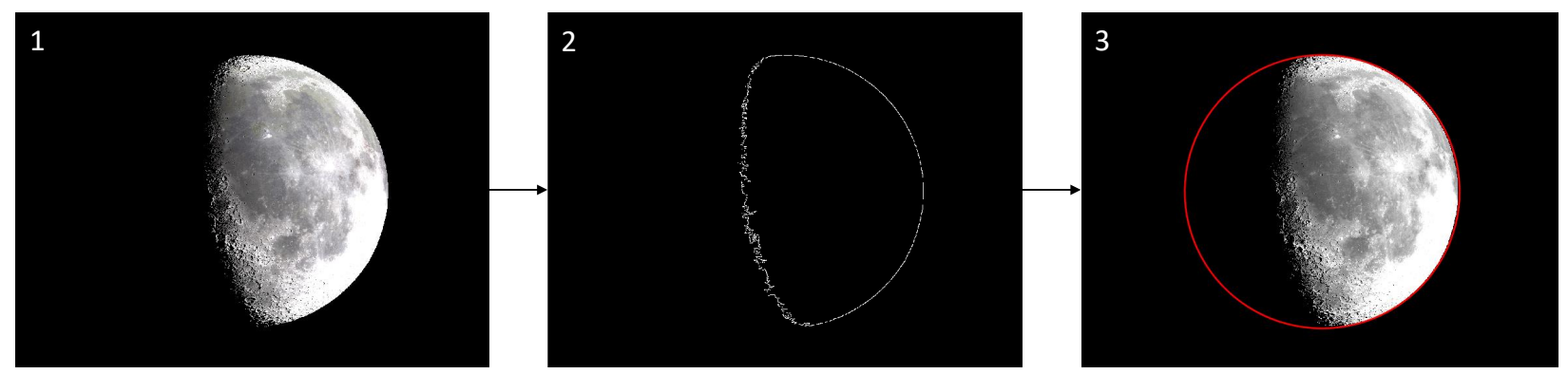

Fig. 7 Lunar images processing: 1) Image Acquisition; 2) Edge Finding; 3) Full Disk Estimation.

\section{E. Navigation Outputs}

The images simulator described in SectionV.Chas been employed to generate 100 images during the LUMIO halo orbits to test the horizon-based optical navigation shown in Section V.D The optical navigation algorithm reports a total position error (norm of position error vector) always below $264.82 \mathrm{~km}$, with the $68 \%$ of the outcomes bounded in 85 $\mathrm{km}$ total error. An extended Kalman filter for navigation has been implemented, and a margin of $37.35 \%$ has been applied to the maximum total error as input to the filter. This is required for the novelty of the navigation algorithm. The navigation filter must take into account the requirements in Table 2, and the filter requirements to satisfy, tunings, and performances are shown in Figure 8 . The halo periods are delimited by the dashed vertical lines. The station keeping planning in terms of maneuvers execution and cut-off times for accurate navigation are shown in Figure $8 \mathrm{a}$, where three station keeping maneuvers are planned for each engineering orbit and none is present in the scientific orbit. Accurate navigation is required 24 hours in advance of the maneuvers (cut-off time). To comply with this, the acquisition frequency has been fine tuned employing three different values, which are $16.7 \mathrm{mHz}$ (High Frequency - HF), $1.67 \mathrm{mHz}$ (Medium Frequency - MF), and $0.277 \mathrm{mHz}$ (Low Frequency - LF), and their employment is shown in Figure $8 \mathrm{~b}$. The high acquisition frequency is used before the cut-off time for SK, while measurements are acquired with the medium frequency during nominal operations in the engineering orbit. For the scientific orbit, measurements are acquired with the low frequency to relax the processing required for navigation and dedicate it to scientific images processing. The $3 \sigma$ position bounds for each component are shown in Figure $8 \mathrm{c}$. The outputs of the navigation filter for LUMIO are compliant with requirements in Table 2

\section{Conclusions}

In this paper we described the SysNova LUCE challenge and analyzed in details LUMIO, one of the four missions that were part of the challenge. LUMIO observes meteoroid impacts on the Lunar farside in order to study the characteristics of meteoroids and to improve the meteoroid models. The mission utilizes a $12 \mathrm{U}$ form-factor CubeSat which carries the LUMIO-Cam, an optical instrument capable of detecting light flashes in the visible spectrum to continuously monitor and process the data. The mission implements a novel orbit design and latest CubeSat technologies to serve as a pioneer in demonstrating how CubeSats can become a viable tool for deep space science and exploration. In this paper we focused on the autonomous operations strategy that is required to guarantee the success of the mission, also looking into 


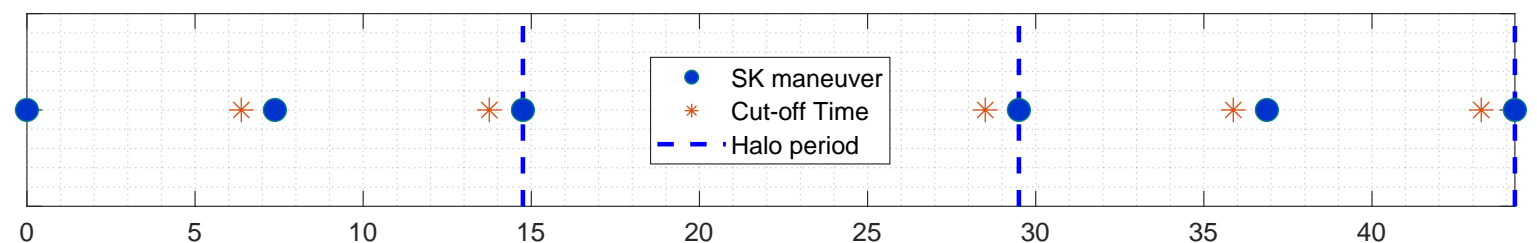

(a) Station Keeping maneuvers planning and execution times. Three maneuvers are commanded each engineering orbit while none is present in the scientific orbit. The cut-off time for accurate navigation is 24 hours in advance of the maneuvers execution.

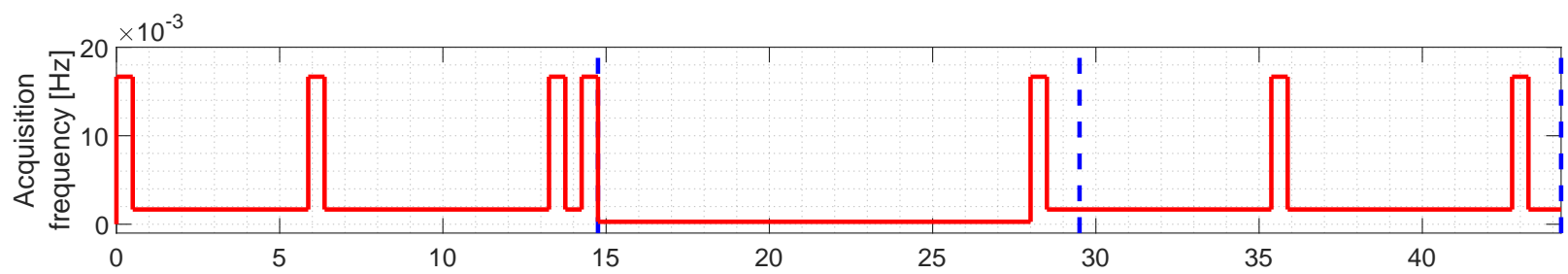

(b) Measurements acquisition frequency tuning. During the engineering orbit, the HF is employed at the cut-off time for SK maneuvers, otherwise the MF. During the scientific orbit, measurements for navigation are acquired with the LF, except when higher frequencies are required for station keeping duties.

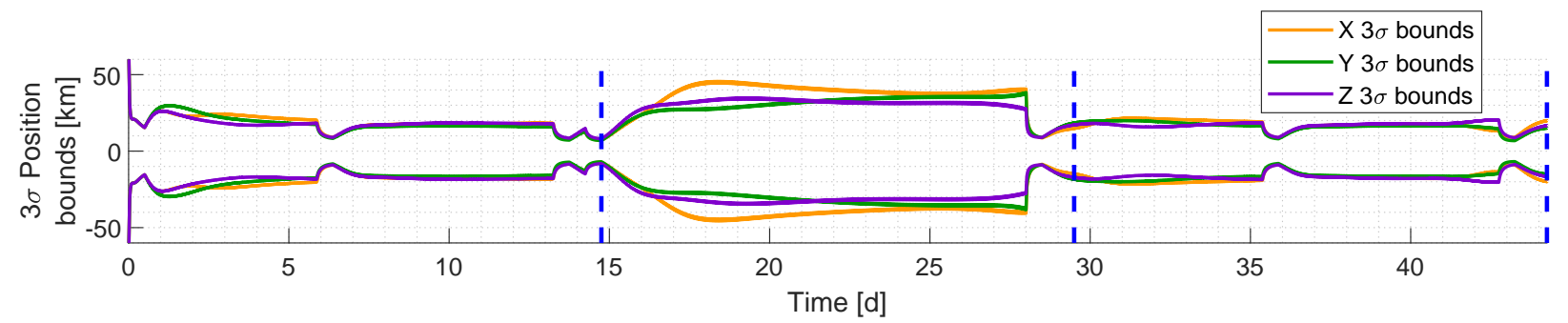

(c) Outputs of navigation filter in terms of position $3 \sigma$ covariance bounds. The spacecraft position is always determined within $30 \mathrm{~km}$ during the engineering orbit and $50 \mathrm{~km}$ during the scientific orbit.

Fig. 8 (a) Station keeping planning, (b) acquisition frequencies and (c) navigation filter outputs in terms of position components for LUMIO.

autonomous navigation. We showed how LUMIO will be capable of estimating its position and calculating navigation information without help from ground. LUMIO, as presented in the paper, will not rely on common navigation and control strategies and this will make it a precursor for future autonomous missions towards planetary bodies.

\section{Acknowledgments}

This work has been funded through the ESA GSP program, Contract No. 4000120225/17/NL/GLC/as, as part of the SysNova LUCE challenge. LUMIO won ex-equo the challenge and was awarded a session in the ESA concurrent design facility to verify and refine the mission concept. We are therefore thankful to the European Space Agency for the support received.

\section{References}

[1] R. Walker, J. Vennekens, R. Fisackerly, J. Carpenter, I. Carnelli, "LUnar CubeSats for Exploration (LUCE) mission concept studies", 6th Interplanetary CubeSat Workshop, Cambridge, UK 30/05/2017

[2] R. Walker, D. Koschny, C. Bramanti, I. Carnelli, ESA CDF Study Team, "Miniaturised Asteroid Remote Geophysical Observer (M-ARGO): a stand-alone deep space CubeSat system for low-cost science and exploration missions", 6th Interplanetary CubeSat Workshop, Cambridge, UK 30/05/2017

[3] Sheikh S. I., et al. "Spacecraft Navigation Using X-Ray Pulsars", Journal of Guidance, Control, and Dynamics, Vol. 29, No. 1 (2006), pp. 49-63. https://doi.org/10.2514/1.13331 
[4] Mortari D., \& Conway D. "Single-point position estimation in interplanetary trajectories using star trackers", Celestial Mechanics and Dynamical Astronomy, May 2017, Volume 128, Issue 1, pp 115-130. https://doi.org/10.1007/s10569-016-9738-4

[5] Christian J. A., \& Robinson S. B. (2016). "Noniterative Horizon-Based Optical Navigation by Cholesky Factorization", Journal of Guidance, Control, and Dynamics, Vol. 39, No. 12 (2016), pp. 2757-2765. https://doi.org/10.2514/1.G000539

[6] Acton Jr C., "Ancillary data services of nasa's navigation and ancillary information facility", Planetary and Space Science 44(1), 65-70 (1996). DOI:10.1016/0032-0633(95)00107-7

[7] Acton Jr C., Bachman N., Semenov B., Wright E., "A look towards the future in the handling of space science mission geometry". Planetary and Space Science 150, pp. 9-12 (2018). DOI:10.1016/j.pss.2017.02.013

[8] Canny J. "A Computational Approach to Edge Detection", IEEE Transactions on Pattern Analysis and Machine Intelligence, PAMI-8(6) (1986), pp. 679-698. https://doi.org/10.1109/TPAMI.1986.4767851

[9] Vittorio Franzese, Pierluigi Di Lizia, and Francesco Topputo. "Autonomous Optical Navigation for LUMIO Mission", 2018 Space Flight Mechanics Meeting, AIAA SciTech Forum, (AIAA 2018-1977) https://doi.org/10.2514/6.2018-1977

[10] Proximity-1 Space Link Protocol — Rationale, Architecture, And Scenarios, CCSDS Green Book, CCSDS 210.0-G-2

[11] D. A. D. Tos and F. Topputo, "On the advantages of exploiting the hierarchical structure of astrodynamical models," Acta Astronautica, vol. 136, pp. 236-247, 2017.

[12] D. A. Dei Tos and F. Topputo, "Trajectory refinement of three-body orbits in the real solar system model," Advances of Space Research, vol. 59, no. 8, pp. 2117-2132, 2017.

[13] SRE-PA \& D-TEC staff, "Margin philosophy for science assessment studies," 2012. 